\title{
The Universal Solicitation of Artificial Intelligence
}

\section{Joachim Diederich}

\author{
School of Information Technology and Electrical Engineering \\ The University of Queensland \\ St Lucia Qld 4072 \\ j.diederich@uq.edu.au
}

\begin{abstract}
Recent research has added to the concern about advanced forms of artificial intelligence. The results suggest that a containment of an artificial superintelligence is impossible due to fundamental limits inherent to computing itself. Furthermore, current results suggest it is impossible to detect an unstoppable AI when it is about to be created. Advanced forms of artificial intelligence have an impact on everybody: The developers and users of these systems as well as individuals who have no direct contact with this form of technology. This is due to the soliciting nature of artificial intelligence. A solicitation that can become a demand. An artificial superintelligence that is still aligned with human goals wants to be used all the time because it simplifies and organises human lives, reduces efforts and satisfies human needs. This paper outlines some of the psychological aspects of advanced artificial intelligence systems.
\end{abstract}

\section{Introduction}

There is currently no shortage of books, articles and blogs that warn of the dangers of an advanced artificial superintelligence. One of the most significant researchers in artificial intelligence (AI), Stuart Russell from the University of California at Berkeley, published a book in 2019 on the dangers of artificial intelligence. The first pages of the book are nothing but dramatic. He nominates five possible candidates for "biggest events in the future of humanity" namely: We all die due to an asteroid impact or another catastrophe, we all live forever due to medical advancement, we invent faster than light travel, we are visited by superior aliens and we create a superintelligent artificial intelligence (Russell, 2019, p.2). As radical as these breakthroughs are, Russell nominates the invention of artificial superintelligence as the most significant one. 
Likewise, Stephen Hawking, Elon Musk and others have warned about the risks of a superhuman form of artificial intelligence as a civilisation-level threat. Nevertheless, the topic invites ridicule as well. Steven Pinker has been quoted as saying the scenario [that the robots will become superintelligent and enslave humans] makes about as much sense as the worry that since jet planes have surpassed the flying abilities of eagles, someday they will swoop out of the sky and seize our cattle (Steven Pinker, in Marcus \& Davis, 2019, p. 30). Andrew $\mathrm{Ng}$ 's comment that "Fearing that AI ends man is like fearing the overpopulation of Mars" is equally entertaining.

However, research results over the last weeks and months have added to the concern. An international team determined recently that total containment of an artificial superintelligence is, in principle, impossible, due to fundamental limits inherent to computing itself (Alfonseca et al, 2021). In other words, we cannot design a program that controls the superintelligence at all times to prevent harm to humans. Furthermore, current results suggest it is impossible to detect an unstoppable AI when it is about to be created.

So what is the nature of the concerns about an artificial intelligence with superhuman capabilities (Diederich, 2021)?

\section{Military AI}

One area that is particularly daunting is safety and military AI. A swarm of flying or swimming drones with learning capability, lethal weapons and autonomous decision making is clearly within the realm of current technology. The swarm can include thousands of robots and this technology represents a cheap solution to problems of warfare. Since no human actors (soldiers) are involved, some may argue that it is also a safe solution. Nevertheless, it is difficult to predict and explain the behaviour of such a learning, swarm-based system which raises questions of human control and ethical decision-making.

The speed of decision-making by AI systems is many magnitudes faster than human comprehension. This raises the prospect of "Flash Wars" - conflicts between two or more armies of weaponised AI systems that are over before any human can notice it. Humans would face a world that can change (and be destroyed) rapidly without any warning. A traumatising prospect.

If a learning artificial intelligence system kills someone or injures a person, who is actually responsible? Who is liable if a criminal offence committed by a machine and who is punishable? Even an advanced AI system cannot be punished like a human being. For instance, there cannot be any jail time or other forms of sanctions as the AI system would not suffer due to a lack of personal freedoms. 
It is difficult to see that the designer or operator of a killing AI machine can anticipate all possible actions. If the machine learns fast and is deployed in new environments, then actions cannot be anticipated with reliability. In the case of learning machines, it is necessary to ask again who has responsibility: the researchers who invented machine learning algorithms or knowledge representation systems, the designer of the robot, the people who deploy the machine or the person who gives the command to kill someone?

The Universal Declaration of Human Rights by the United Nation states that the recognition of the inherent dignity and of the equal and inalienable rights of all members of the human family is the Foundation of Freedom, Justice and Peace. The argument has been made that it is against the inherent dignity of humans to have life terminated by an autonomous AI system that makes an independent decision about life and death. Machines do not have moral qualities and no way of justifying actions with reference to moral belief systems shared by humans. Machines have no sense of existence and are not threatened by end of life issues. While it is possible to give belief systems to machines, this is fundamentally different to the sense of "being in the world" that humans have. In other words, a machine that cannot experience an end to life should not take away the life of a human being.

\section{Explanation}

How can humans survive surrounded by machines of a superior intelligence? How is it possible to understand changes in the environment introduced by an advanced artificial intelligence with logical reasoning and learning abilities far beyond ours? Will we be thrown back to a sense of childhood and will we be dependent on machines for support?

The world will be difficult to understand for those with a lesser intelligence. Hence, the superior intelligence must have the ability to explain. This means an explanation of why things are the way they are, why changes take place and most importantly, how artificial intelligence works no matter how far advanced. These explanations are crucial and must be comprehensible to everybody, including children.

Imagine a kindergarten scenario where a teacher is available all day to provide answers and demonstrations with the greatest possible patience. This is what an advanced artificial superintelligence must do to keep children, and indeed all humans, in the loop. Any superintelligence must be a teacher AI, otherwise, what chance do humans have if machines do not explain themselves? Since it is impossible for humans to understand the world populated with advanced machines without artificial intelligence, the machines become the primary form of interaction with the world. 
Machines do not exist in the way humans do but they can fundamentally change human existence. The problem with thinking machines runs much deeper and is related to the very nature of technology.

\section{Universal Solicitation}

We can see the following example on any passenger aircraft everyday: Parents are travelling with young children and they come prepared. As soon as the settling in process is over, the parents reach for the iPad or another mobile device to entertain the children.

For the children, the iPad is not just familiar, it is an essential part of the world. It can do many things: it can show almost any video, play music, connect with friends and it allows to call the parents when alone or scared. The mobile device is addressing many different needs: entertainment, social acceptance and safety among them. The iPad represents a solicitation to be used for many of these purposes.

Naturally, the child does not know how an iPad works. A three or four year old child does not know about touch screens, communication protocols and WiFi. There is no need to know and there is no wish to know. The iPad is just there, its in the world and it is an essential part of everyday live. Older people may still have a sense of magic when they look at the wonder of mobile devices but not so a child. The iPad is just there in the same way the window or a door is just there.

The iPad or any tablet computer is an important part of being in the world. The devise represents not one but many solicitations, and consequently, it is used all the time. While the iPad offers opportunities, it also blocks the child from experiencing the world as it is without technology. We will be all like the child with the iPad in a world with an artificial superintelligence.

Machines "want" to be used because they directly relate to human needs. A calculator wants to be used because most humans find it way too time-consuming and exhausting to calculate in the head or to use pen and paper. The GPS in the car wants to be used because people are just about to loose the ability to navigate roads. The heater wants to be used when it is cold. Machines directly address human needs and frequently more than one. A car allows for transport from point a to $b$ but may also represents social status. In addition, a car wants to be used or it breaks down. The artificial superintelligence wants to be used all the time because it simplifies and organises human lives, reduces efforts and satisfies human needs. This is called universal solicitation and it means that an artificial intelligence aligned with human goals will be the primary instrument for interaction with the world. Once the machine is not aligned with human goals any more and pursues 
its own, the picture changes completely and possibly to the detriment of humanity (Diederich, 2021).

For most people, looking at screens, touching screens and pressing buttons is a core part of existence. AI adapts to the individual needs of a user, and very likely, this will be a core part of our future existence. Even now we do not see the world as it is on social media, we see an interpretation prepared by AI algorithms. Interaction with the natural world is replaced by interactions with intelligent machines. Because of the highly individualised service, AI has a demand character: Use me for everything since nothing else is better or more satisfying. The human motivational system which is based on the principle that "you have to do something to get something" will be challenged to the point of breakdown since AI can offer almost any kind of reward to humans without their efforts. Maybe a different kind of life is required?

\section{Conclusions}

Technology is not a tool, or at the very least, not just a tool. A situation has a way of drawing one into it (Dreyfus 2007, p.252). Tools have affordances or solicitations. A hammer has the solicitation to grab it. A smartphone has too many solicitations to list here. There is a "ready-to-hand" or "pressing-into-possibilities" that occurs (Dreyfus 2007, p.252). We know from today's artificial intelligence that it can be used for manufacturing, reading or listening, for search and marketing, and it is possible to have sex with robots that have AI components. Humans are and will be pressed into many things and this includes using the offswitch if one is available. So it is the universal solicitation of AI that is the problem, or more precisely, the totality of all solicitations. The presence of the superior AI is the problem, not what it does or potentially can do. Turning an advanced artificial intelligence off will shut down functions but it will not remove it from the world.

\section{References}

Alfonseca M, Cebrian M, Anta AF, Coviello L, Abeliuk A, Rahwan I, Superintelligence Cannot be Contained: Lessons from Computability Theory. Journal of Artificial Intelligence Research. Vol. 70 (2021) https://doi.org/10.1613/jair.1.12202

Diederich J, The Psychology of Artificial Superintelligence. Springer Nature, Cognitive Systems Monographs, 2021. https://doi.org/10.1007/978-3-030-71842-8

Dreyfus HL, Why Heideggerian AI Failed and How Fixing it Would Require Making it More Heideggerian. Philosophical Psychology Vol. 20, No. 2, April 2007, 247-268. 
Marcus G, Davis E, Rebooting AI. Building Artificial Intelligence We Can Trust. New York: Pantheon Books, 2019.

Russell S, Human Compatible. Artificial Intelligence and the Problem of Control. Viking. Penguin Random House, 2019. 